\title{
Analysis of the Structure of a Hard Metal: A Simple Method of Relating Properties to Stereological Structures
}

\author{
Antônio Carlos Buriti da Costa*, Angelus Giuseppe Pereira da Silva ${ }^{\text {b }}$ Uílame Umbelino Gomes ${ }^{\mathrm{a}}$ \\ ${ }^{a}$ Universidade Federal de Campina Grande, Campina Grande - PB, Brazil \\ ${ }^{\mathrm{b}}$ LAMAV, Universidade Estadual do Norte Fluminense, Campos - RJ, Brazil \\ ${ }^{\mathrm{C}}$ Dpto. Física, Universidade Federal do R. G. do Norte, Natal - RN, Brazil
}

Received: November 23, 2003; Revised: March 13, 2005

\begin{abstract}
Computer simulation of the hard phase of WC-based hard metals is not common in scientific literature. This phase is formed by carbide grains embedded in a metallic matrix. In the equilibrium state, the grain has an equilateral prismatic form with beveled edges. It is nevertheless possible to model stereological aspects of this phase, using a Monte Carlo simulation assuming a prism structure without beveled edges. From the grain's height to width ratio, physical parameters such as grain area and intercept lengths can be calculated. The goal of this work is to present a simple method to obtain this ratio for metallographic samples. One of the results is to establish a relationship between the intercept length and the true size of the grain, which facilitates better understanding of this material's structure.
\end{abstract}

Keywords: hard metal, aspect ratio, computer simulation, metallography

\section{Introduction}

A hard metal is a composite material consisting of the hard phase embedded in a binder phase. The hard phase consists of grains of tungsten carbide (WC) cemented to the binder phase consisting of cobalt or nickel.

Composite materials exhibit properties that are a combination of properties of its components. In these materials, improvements have been achieved in terms of increased hardness with the inclusion of other carbides, or even inhibited grain growth, and these contribute to decrease in impact resistance. The hardness and impact resistance are mainly controlled by the composition of the phases. These properties are crucial for use of the composite as a cutting tool, which is its main area of application. Depending on the specific application, these properties can be adjusted by varying the relative composition of the phases. This is important because the harder a material the lower its impact resistance. Nevertheless, refinement of WC grains increases impact resistance of the material without affecting hardness ${ }^{1-5}$.

Figure 1 shows the microstructure of a WC-based hard metal cemented by nickel. The grains, when intercepted by random planes, are in the shape of very well defined sections with three, four or five sides in general.

This work characterizes stereological aspects of the hard metal through computational simulation, using the Monte Carlo method ${ }^{6}$. The model used in the simulation allows us to calculate the area of the generated random sections of intercepted grains, the intercept lengths, and types of intercept sections. In the case of mean intercept length measurements, a relationship between the known height to width ratio of the grain, defined as aspect ratio, is calculated. The simulated variations in the figure type as a function of aspect ratio allows us to identify the aspect ratio of metallographic samples in a simple way ${ }^{4}$.

\section{The Simulation Method}

Figure 2 displays a straight prism with a triangular equilateral base, used to simulate grains of WC. The ratio of the height $h$ to the side length $L$ defines the aspect ratio $\alpha$. Emphasis is given to the generation of random data, using the Monte Carlo method. The following procedure was adopted in the simulation of the hard metal:

a) In a Cartesian reference system, the values of $h$ and $L$ are used to calculate the coordinates of the vertices, the surface area $S$, $S=(0.867+3 . \alpha) . L^{2}$, and the volume $V, V=0.433 . \alpha . L^{3}$;

b) The equivalent spherical size of the prism is calculated as being the diameter $\Phi$ of a sphere that has the same volume;

c) The prism is rotated randomly around the $X, Y$ and $Z$ axes, with angles between 0 and $2 \pi$;

d) The projected heights $\mathrm{x}_{\max }-\mathrm{x}_{\min }, \mathrm{y}_{\max }-\mathrm{y}_{\min }$ and $\mathrm{z}_{\max }-\mathrm{z}_{\min }$ (see Figure 3 ) are determined. The mean values of the heights projected along 3 axes should be practically the same for a large number of prisms. This serves as a test of the randomness of the data;

e) A point on the $Z$ axis is randomly chosen within the overall prism height and the prism is cut with a plane, parallel to $X Y$ that passes though this point. This generates the intersection figure (section);

f) The area of the figure (which may have 3, 4, or 5 sides) is calculated;

g) An angle between 0 and $2 \pi$ is randomly chosen and the vertices of the intersection figure are rotated around the $Z$ axis by this angle;

h) The extreme values of the $Y$ coordinates of the figure are determined;

i) A $Y$ value between these extremes is randomly chosen. The length of the intercept parallel to the $X$-axis that cuts the figure at this $Y$ value is determined; and

j) The mean value of the intercept length for the whole sample is calculated and the number of 3 -, 4 -, and 5 - sided figures are counted. The resulting value of the mean intercept length is compared with that predicted $I_{T}$ from stereological theory ${ }^{7}$ : $I_{T}=4 . V / S$. 


\section{Results and Discussion}

The model was exhaustively tested for the perfect random nature of the data. Random error is subtle and hence difficult to detect. Reference 7 suggests a series of tests for checking the random properties of the data. It is suggested that at the time the solid on which one is working is sectioned, a sphere bounded by the solid should also be sectioned. The fact that the distributions of intercept and sectional area of the sphere are widely known, if results obtained are not the same as the patterns for the sphere, the data is not random.

Two tests, based on the above principle were performed to ensure maximum randomness. The first test used the simulated mean intercept length. This value should be compatible with the average intercept predicted by the theorem cited above. In case $I_{T}=2.46 \mu$.

The second used the simultaneous comparison of the "heights" projected by the prism in the coordinated $X, Y$ and $Z$-axis. These projected heights were calculated for each rotated prism. If the rotations are in fact random, the three mean values should be the same, within an intrinsic error characteristic of the Monte Carlo method; because the value of the mean projected height should be independent of direction.

The values produced by the simulation should be compared with the previous results to increase accuracy of modeling. For the specific case of prismatic grains of hard metal, the only reference is Dehoff et al. ${ }^{7}$ However, their results could not be compared with those of this work, because they considered only truncated base prisms. Nevertheless, in reference 7 no credible test for the results was mentioned.

Table 1 shows the average values of the mean projected heights and intercept lengths, and their standard deviations over 5 simulations. Each simulation considered 1000 prisms with the equivalent grain size of $5 \mu \mathrm{m}$ and aspect ratio of $1 / 2$. This value of aspect ratio was taken from reference 8 , which uses an aspect ratio of about 0.43 . The values of mean intercept length and projected height are presented with a $10^{-2}$ computational accuracy.

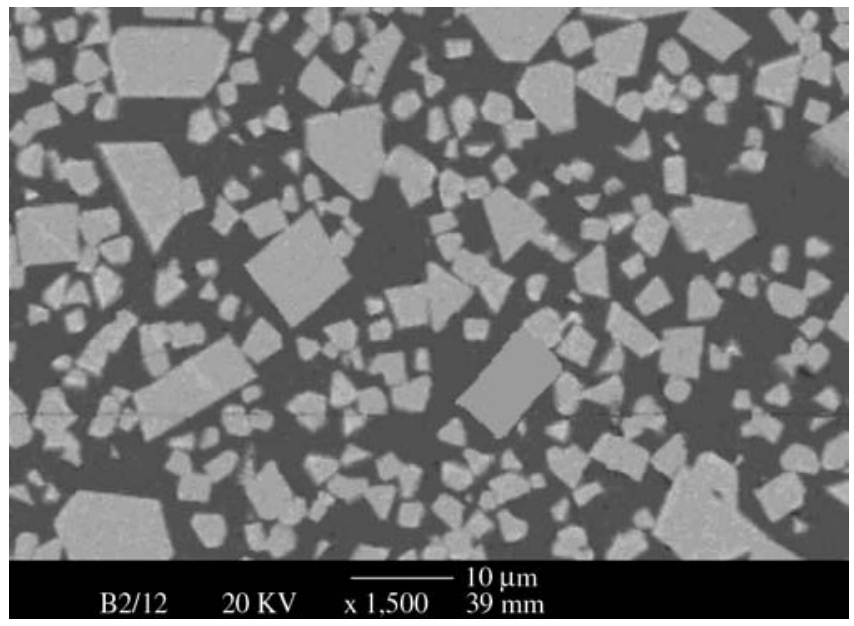

Figure 1. Cross-section of a WC-30 wt.(\%)Ni composite.
Mean measured intercept is compatible with the theoretica intercept. The standard deviation is small. The statistical error for intercept $\Delta I, \Delta I=\left|\left(I_{T}-I_{M}\right) .100 / I_{T}\right| \%$, is less than $0.2 \%$, which is an excellent result. The mean projected heights are practically identical. This demonstrates the randomness of the data.

In all measurements of grain size taken in metallographic planes, the number of measured grains is an important factor, because it determines the precision of the measurement. It is natural to expect that, when dealing with measurements whose individual values are distributed randomly. As the number of measurements increases the mean value converges to a certain value. The closer the mean value is to this converging value more reliable will be the measurement. It is important to determine the number of measurements that should be taken. As the measurements of grain size are, in general, very difficult, involving the preparation of the sample, obtaining a series of images and the measurement itself, it is preferable that the number of measurements be the least possible. A compromise between a minimum number of measurements and a minimum acceptable level of precision has been used.

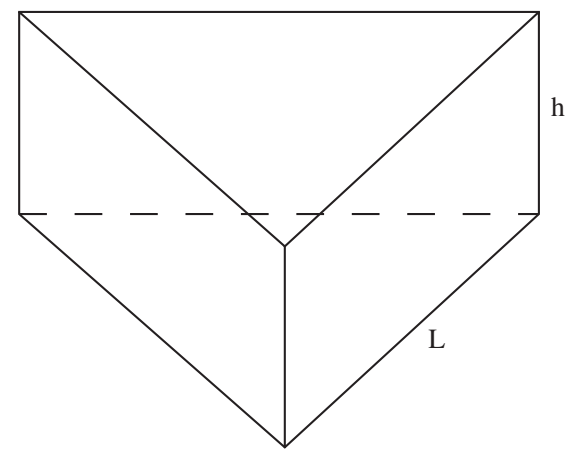

Figure 2. Prism with an equilateral triangle base used to simulate the grains of tungsten carbide (WC)

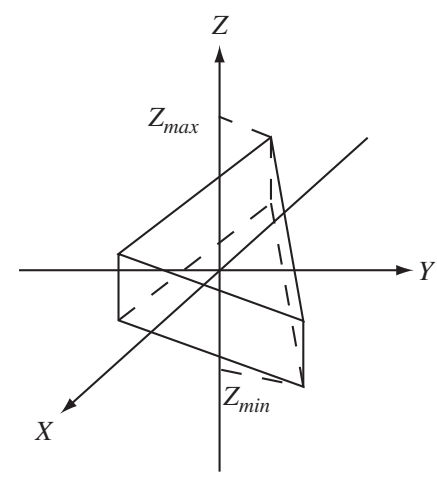

Figure 3. Definition of the projected height of the prism in the $Z$ direction. The same can be done in the directions $X$ and $Y$.

Table 1. Mean values, standard deviation and error in five simulations for 1000 prisms of equivalent grain size of $5 \mu \mathrm{m}$ and aspect ratio of $1 / 2$. The theoretical intercept $I_{T}$ is $2.46 \mu \mathrm{m}$.

\begin{tabular}{lccccc}
\hline & $\begin{array}{c}\text { Mean measures } \\
\text { intercept, } \bar{I}_{M} \\
\mu \mathrm{m}\end{array}$ & $\begin{array}{c}\text { Theoretical } \\
\text { intercept, } I_{T} \\
\mu \mathrm{m}\end{array}$ & $\begin{array}{c}\text { Statistical error, } \\
\text { ( }\end{array}$ & \multicolumn{2}{c}{ Mean values of projected heights in axis } \\
$Z$
\end{tabular}


In practical experiments, the number of measured grains varies from author to author, as there are no standards regarding this. In the references used, the number of measurements varied from a little over two hundred to two or three thousand grains. It seems that, in practice, the number of measurements is dictated by the extent of effort and not by the level of trust in the mean value. Publications referring to this aspect were not found. This type of study on real samples would require considerable effort. Computational simulation of the structure allows us to conduct such a study without much effort.

The graph shown in Figure 4 indicates how the mean values of intercept length vary (close to its standard deviation) and their respective statistical deviation. To model the curves, five simulations were made for each sample of simulated grains. It is clearly seen that as expected, the error decreases as the number of grains increases. However it decreases differently for each type of parameter. For measurements of mean intercept with 400 measured grains, the statistical deviation is around $2 \%$ or $3 \%$, which is the characteristic level of statistical deviation for the Monte Carlo method. For greater confidence, simulations with 500 or more grains are recommended. A statistical analysis, similar to that applied to the intercept length, was applied to populations of 3 -, 4 -, and 5 - sided figures, as shown in Figures. 5a, 5b and 5c, and Table 2.

Roebuck et al. ${ }^{8}$ determined the aspect ratio of the WC grains from observations of the grains located on the pore surface of the hard metal. Dattrataya ${ }^{9}$ and Kim et al. ${ }^{10}$ determined the aspect ratio from measurements of the aspect ratio of cut figures in metallographic planes. A certain fraction of the intersection figures have four sides. Usually such figures are trapezoidal. The aspect ratio of these figures was defined as being the ratio between the longest parallel side and the height of the trapezium. When the height of the trapezium was longer, the inverse of this ratio was adopted.

This measurement is very tedious, because it is essential to identify a large number of these sections and measure two lengths for each section. The aspect ratio of the prism will be the largest value of aspect ratio of the intersection figures, because this value corresponds to the cut of the prism for a plane perpendicular to the base plane with the cut section parallel and very close to one of the lateral faces of the prism. It should be emphasized that these authors defined the aspect ratio of the prism as being the ratio between the base and the height of the prism. This is exactly the opposite of the definition that was adopted in this work.

The aspect ratio of the prism can be determined using a simpler measurement technique than the one used by the authors cited above.

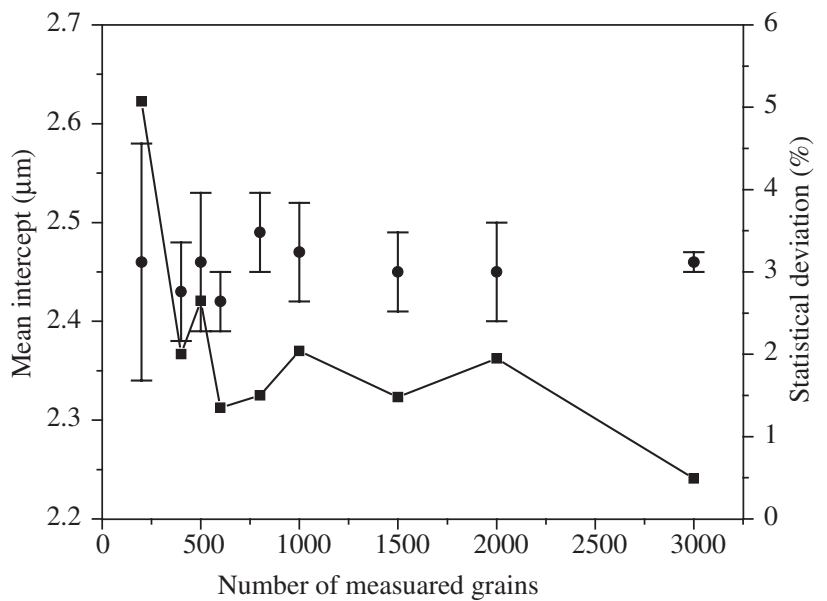

Figure 4. Mean intercept length and statistical deviation $v s$. the number of measured grains.
It is sufficient to just count the number of three or four sided illustrations that are intercepted.

It is easy to imagine that triangular sections are much more easily formed in prisms of high aspect ratio, while sections of four sides are more easily produced in prisms of low aspect ratio. This

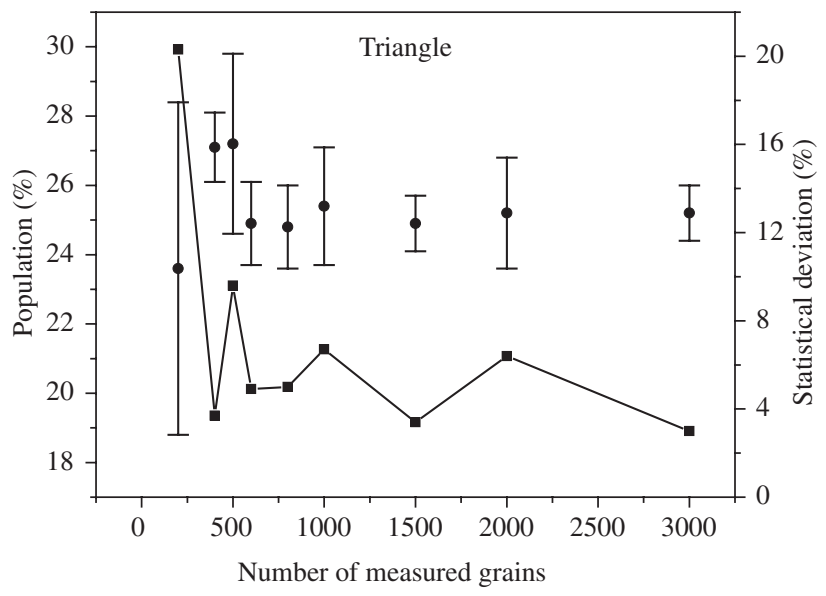

(a)

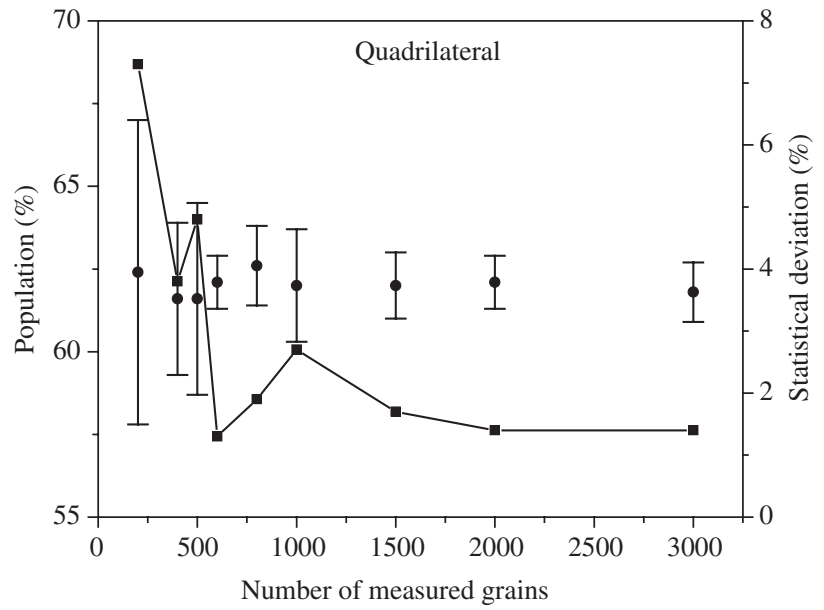

(b)

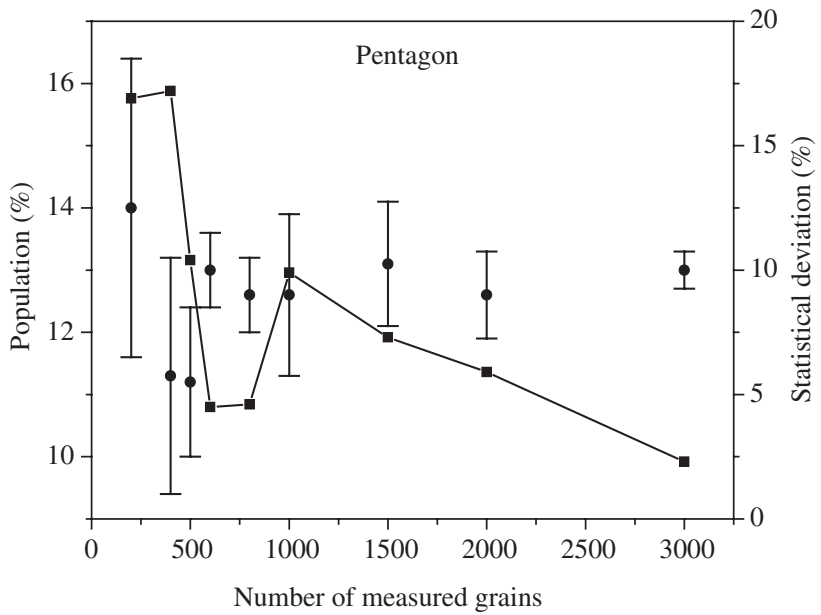

(c)

Figure 5. Population of triangular, quadrilateral and pentagonal cut sections and the statistical deviation pattern $v s$. the number of measured grains. 
Table 2. Partial and final mean values, deviations and errors in 10 simulations of 1000 prisms of equivalent grain size of $5 \mu \mathrm{m}$ and aspect ratio $1 / 2$.

\begin{tabular}{lcccc}
\hline & $\begin{array}{c}\text { Mean intercept } \\
(\mu \mathrm{m})\end{array}$ & 3 sides figure $(\%)$ & 4 sides figure $(\%)$ & 5 sides figure $(\%)$ \\
\hline Mean & 2.47 & 6.69 & 6.70 & 6.70 \\
Standard dev. & 0.06 & 0.01 & 0.01 & 0.01 \\
Error $(\%)$ & 2.37 & 0.17 & 0.20 & 0.16 \\
\hline
\end{tabular}

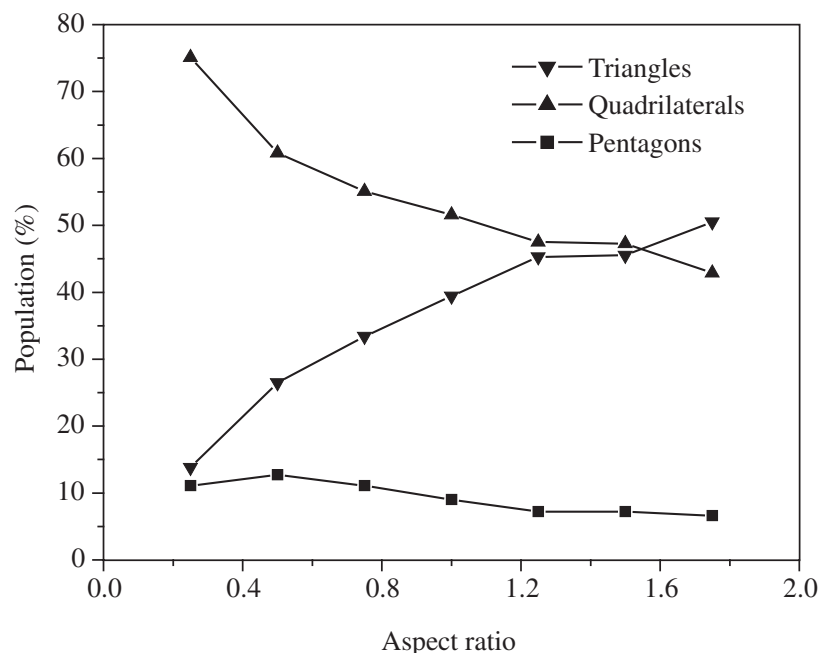

Figure 6. Number of 3 -, 4 - and 5 - sided intersection figures $v s$. the aspect ratio of the prism.

was confirmed by counting the number of three, four and five-sided figures in simulations of prisms with different aspect ratios. For each aspect ratio, 1000 prisms were simulated. The graph in Figure 6 shows the result.

Prisms with aspect ratios varying from $1 / 4$ to $13 / 4$ were simulated. The number of five-sided intersection figures changes only slightly over the whole interval. As the aspect ratio increases to $11 / 4$, the population of four-sided figures falls at almost the same rate as the population of three-sided figures grow. Above this value of aspect ratio, the population of these two types of figure intersections remains almost constant. This qualitative behavior was expected. However the simulation yielded numbers which provided a simple method for determining the aspect ratio of the grains of WC. It is sufficient to count the number of three or four sided figures in the microstructure and compare with the numbers from this graph. This method however can only be applied to prisms with aspect ratios less than $11 / 4$, because above this value, the number of three and four sided figures is virtually constant, and the measurement errors make them unreliable.

It is important to emphasize that these results do not depend on the equivalent size of the prism. The method is also useful for mixtures of grains of different equivalent sizes ${ }^{4}$. In spite of this, the applicability of such a method is quite debatable due to some factors listed below. All these factors also affect the method used by Dattrataya and $\mathrm{Kim}^{9,10}$ :

a) All the grains of WC should be well formed (in the equilibrium form). They cannot be in the process of being created, because this would generate sections with other formats;

b) The grains cannot have a truncated base, because this would generate intersection figures of other types;

c) All the grains should have the same aspect ratio, because grains with different aspect ratios would distort the shape of the curve presented in Figure 6. There is no record of any study about the existence of grains with different aspect ratios in the micro- structure of a hard metal, i.e. there is neither confirmation nor negation. However, it could happen. Kim et al. ${ }^{10}$, for instance, said that the aspect ratio depends on the amount of carbon in the alloy. The carbon content can vary throughout the microstructure to have grains with different aspect ratios; and

d) All the sections should be well delineated to be identified properly. This depends on the quality of sample preparation and the quality of the microstructure.

The value of the mean intercept is related to the aspect ratio and the side length $L$ by the equation $I_{T}=\sqrt{3} . \alpha \cdot L(0.87+3 . \alpha)$, deduced from the previous relationships. This result is important, since it implies that if the mean intercept length and the aspect ration are known, the dimensions of the WC grain can be determined.

\section{Conclusions}

- It is possible to calculate the mean intercept of a hard metal structure and the size of a single grain, using as few as 500 grains; and

- Aspect ratios of WC grains can be determined much faster by simply counting the number of 3 - or 4 - sided intersection figures, compared to methods previously used.

\section{Acknowledgments}

The authors are grateful to UFRN and UFCG for their financial support and also to Dr. Tom Dumelow and Antônio Carlos Buriti da Costa Filho for helping with the text revision.

\section{References}

1. Gurland J, Knox D. A Historical Review of Two Related Technologies. In: Proceedings of the Second International Conference on Tungsten and Refractory Metals; 1994 October 17-19; VA, USA. VA: Mclean; 1994. v. 1 , p. $220-227$.

2. Pastor H. A General aspects and limits of conventional hard metal. In: Proceedings of the 1996 European Conference on Advances in Hard Materials Production; 1996 September 13-15; Stockholm, Sweden, v. 1, p. 3-11.

3. Brookes K. Hard metals and other hard materials. 3 ed. International Carbide Data, Hertfordshire, UK, 1998, v. 1, p. 72-88.

4. Da Costa A. Granulometric measures simulation of WC in hard metal alloys. [Unpublished thesis]. Natal, Brazil. UFRN; 2003.

5. Landsperger A, Massa T. Tungsten Consumption Trends in the Mining and Road Construction Industries. In: Proceeding of the $7^{\text {th }}$ International Tungsten Symposium; 1996 Sep 27-30; Dresden, Germany. p. 85-96.

6. Gould H. A introduction to computer simulation methods. 2 ed. Addison Wesley Publishing Company, New York, USA, 1996.

7. Russ J, Dehoff R. Practical stereology. 2 ed. Plenum Press; New York, USA, 1999.

8. Roebuck B, Mc Cormick N, Day A, Bennett E. Measurement of WC Grain Size Distribution. NPL Report CMMT, 1999; A (1).

9. Dattatraya D. A study of liquid phase sintering and micromorphology of tungsten carbide-cobalt alloys. [Unpublished thesis]. Boston, USA. Brown University; 1982.

10. Kim S, Han S, Park J, Kim H. Determination of Thee-Dimensional Grain Size Distribution. by Linear Intercept Measurement In: Proceedings of the 15th International Plansee Seminar, 2001 Nov 21-24, Tirol, Austria, v. 2, p. 294-305. 